\title{
Stationary and dynamical properties of a zero range process on scale-free networks
}

\author{
Jae Dong Noh \\ Department of Physics, Chungnam National University, Daejeon 305-764, Korea
}

(Dated: October 16, 2018)

\begin{abstract}
We study the condensation phenomenon in a zero range process on scale-free networks. We show that the stationary state property depends only on the degree distribution of underlying networks. The model displays a stationary state phase transition between a condensed phase and an uncondensed phase, and the phase diagram is obtained analytically. As for the dynamical property, we find that the relaxation dynamics depends on the global structure of underlying networks. The relaxation time follows the power law $\tau \sim L^{z}$ with the network size $L$ in the condensed phase. The dynamic exponent $z$ is found to take a different value depending on whether underlying networks have a tree structure or not.

PACS numbers: 89.75.Hc, 05.20.-y, 02.50.Ey
\end{abstract}

\section{INTRODUCTION}

A condensation is an intriguing phenomenon observed in nonequilibrium systems [1]. In interacting particle systems, a finite fraction of particles may be condensed onto a single site. Phase separation in driven diffusive systems [2, 3, 4] or jamming in traffic flows [1] is also an example of the condensation. The condensation has been studied within the framework of a zero range process (ZRP) introduced by Spitzer [5]. Those studies revealed that the condensation can be caused by effective attractions between particles or quenched disorder [1, 6, 7, 8, 9, 10, 11]. In the former the condensation is a spontaneous symmetry breaking transition. In the latter it is similar to the Bose-Einstein condensation occurring in the ideal Bose gas [12].

The condensation has been studied mostly on periodic lattices. On the other hand, recent studies show that lattices (networks in general), upon which physical systems are defined, may have more complex structure [13, 14, 15, 16]. Particularly many real-world networks are found to be scale-free. That is to say, they are characterized with the power-law degree distribution

$$
P_{\text {deg }}(k) \sim k^{-\gamma}
$$

where $P_{\text {deg }}(k)$ is the fraction of nodes (or sites) with the degree $k$ (the degree of a node denotes the number of neighbors connected to it) and $\gamma$ is called the degree distribution exponent. This is contrasted to a peaked distribution for periodic regular lattices.

The power-law distribution implies that the SF networks have an inhomogeneous structure. The structural inhomogeneity is an important factor in understanding dynamical processes in SF networks [17, 18, 19, 20, 21, 22]. In random walks, for instance, a diffusing particle visits highly connected nodes more often than others. Moreover diffusion processes are asymmetric. It means that the speed of random walks from a node to another is not equal to that in the reversed direction in general. The asymmetry can be quantified with the socalled random walk centrality [17], which is defined on each node and proportional to its degree. This example shows that each node plays a different role in stochastic processes in inhomogeneous networks. It also suggests that interactions in many particle systems would be important since particle density could be high at some nodes.

In this paper, we study a ZRP on SF networks and investigate the interplay of the dynamics of interacting particle systems and the inhomogeneous structure of underlying networks. The present work is an extension of our recent work presented in Ref. [23], which shows that the structural inhomogeneity gives rise to the novel type of condensation in the ZRP. We will present a detailed review on the results. In addition, we will elaborate a scaling theory for the relaxation dynamics in the condensed phase. Main results are following. The relaxation dynamics has a hierarchical nature; starting from small degree nodes, the relaxation proceeds to larger and larger degree nodes. The relaxation time $\tau$ follows a powerlaw scaling $\tau \sim L^{z}$ with the dynamic exponent $z$ and the network size $L$ in the condensed phase. The relaxation dynamics depends on the global structure of underlying networks so that the dynamic exponent takes a different value depending on whether the networks have a tree structure or not. Usually, the degree distribution is considered to be important in characterizing the dynamics of complex networks. Our result shows that the global structure of networks is an important ingredient in understanding the dynamics.

This paper is organized as follows. In Sec. II we provide a general review on stationary and dynamical properties of the ZRP. Then in Sec. III we introduce the ZRP on SF networks and present its stationary state phase diagram. In Sec. [V]we investigate the relaxation dynamics. We summarize and conclude the paper in Sec. V

\section{ZERO RANGE PROCESS}

Consider a system of $N$ interacting particles on a network of $L$ nodes. The total particle density is given by $\rho=N / L$. Each node $i$ can be occupied by any integral number of particles (no exclusion). With $n_{i}=0,1,2, \cdots$ denoting the occupation number at node $i$, a microscopic configuration is represented with $\mathbf{n}=\left(n_{1}, n_{2}, \cdots, n_{L}\right)$.

A ZRP is specified by jumping rates $\left\{p_{i}(n)\right\}$ and hopping probabilities $\left\{T_{j \leftarrow i}\right\}$. With those, at each node $i$ with $n_{i}>0$, a particle jumps out at the rate $p_{i}\left(n_{i}\right)$ and then hops to a node $j$ with the probability $T_{j \leftarrow i}$. Note that the jumping rate depends 
only on the occupation number at departing nodes. This is an important feature of the ZRP, which makes the exact stationary state probability distribution available [26]. Recently, generalizations with multi-species particles [24] or with multipleparticle hoppings [25] are being considered.

A particle interaction can be incorporated into the jumping rate. If particles are noninteracting, they move independently, hence $p(n) \propto n^{1}$. An attractive interaction can be imposed with the jumping rate function $p(n)$ that grows sub-linearly or decreases with $n$, or a repulsive interaction with a super-linear jumping rate function. In the context of data packet transport, the jumping rate $p_{i}(n)$ corresponds to the number of packets that can be processed by a node $i$ having $n$ packets per unit time. That is, the jumping rate function models the transport capacity.

It is well known that the stationary state property of the ZRP is exactly solvable [1, 7]. For later uses, we list useful formula below. The stationary state probability distribution $P_{\text {stat }}(\mathbf{n})$ is given by

$$
P_{\text {stat }}(\mathbf{n})=\frac{1}{Z(L, N)} \prod_{i=1}^{L} f_{i}\left(n_{i}\right)
$$

where

$$
f_{i}(n)=\prod_{n^{\prime}=1}^{n}\left(\frac{\omega_{i}}{p_{i}\left(n^{\prime}\right)}\right)
$$

for $n \neq 0$ and $f_{i}(0)=1$, and $Z(L, N)$ is a normalization constant. Here, $\left\{\omega_{i}\right\}$ is the solution of coupled linear equations

$$
\sum_{j} T_{j \leftarrow i} \omega_{i}=\sum_{j} T_{i \leftarrow j} \omega_{j} \quad(i=1, \cdots, L) .
$$

Note that these equations are equivalent to those for the stationary state probability distribution for a single random walker problem with the hopping probability $\left\{T_{j \leftarrow i}\right\}$. The left (right) hand side corresponds to the outgoing (incoming) probability flux.

Introducing an auxiliary fugacity variable $z$ [1], one finds that the mean occupation number $m_{i}=\left\langle n_{i}\right\rangle$ is given by

$$
m_{i}=z \frac{\partial}{\partial z} \ln F_{i}(z)
$$

where

$$
F_{i}(z) \equiv \sum_{n=0}^{\infty} z^{n} f_{i}(n)
$$

The series is defined for $|z|<z_{c}$ with $z_{c}$ being the radius of convergence. The fugacity should be determined from the self-consistent equation

$$
\rho=\frac{1}{L} \sum_{i=1}^{L} m_{i}(z)
$$

A ZRP may display the condensation transition. We introduce two instances. A simplest ZRP displaying the condensation might be the one with the jumping rate $p_{i}(n)=$ $\left(1-\delta_{i, i_{0}}\right)+\alpha \delta_{i, i_{0}}$ on a periodic regular lattice with nearest neighbor hoppings [1]. This model has a quenched disorder so that the impurity node $i_{0}$ has a low jumping rate $\alpha<1$. Applying the formalism, one can show easily that the condensation occurs for $\rho>\rho_{c}=\alpha /(1-\alpha)$, where a macroscopic condensate forms at the impurity site with the occupation number $m_{i_{0}}=\left(\rho-\rho_{c}\right) L$, while the occupation number at the normal nodes is given by $m_{i \neq i_{0}}=\rho_{c}$.

The condensation also occurs in a system without quenched disorder. For instance, consider a ZRP on periodic regular lattices with the jumping rate $p_{i}(n)=p(n)=1+b / n$. A straightforward analysis shows that the system is in the condensed phase when $b>2$ and $\rho>\rho_{c}=\rho_{c}(b)$ [1, 6]. In this case, a macroscopic condensate forms at a node selected spontaneously.

As for the relaxation dynamics, an exact theory has not been known yet. However, qualitative features may be understood from a scaling argument [6, 27, 28, 29]. To be specific, we consider the relaxation dynamic of the above example with the jumping rate $p(n)=1+b / n$ in the condensed phase. Being distributed uniformly initially, particles form small clusters at nodes due to a stochastic fluctuation. It is believed that a macroscopic condensate emerges by successive coarsening processes of the small clusters. Those clusters exchange particles, merge into larger ones, and grow until there remains a single macroscopic condensate. This scaling picture predicts the power-law growth of the cluster size in time and the power-law scaling of the relaxation time with the system size as $\tau \sim L^{z}$. It is known that the dynamic exponent $z$ depends on the dimensionality of the underlying lattices and bias in particle hoppings [6, 27].

\section{ZRP ON SF NETWORKS}

A recent study reveals that structural inhomogeneity of SF networks leads to the so-called complete condensation [23]. Being compared to the usual condensation in periodic regular lattices [1], the complete condensation has the features that (i) it occurs at any finite value of the particle density, i.e., $\rho_{c}=$ 0 , and that (ii) the whole fraction of particles are condensed onto a few high-degree nodes. We review these results in this section.

Consider a ZRP on a SF network of $L$ nodes. The network is assumed to have a power-law degree distribution $P_{\operatorname{deg}}(k) \sim$ $k^{-\gamma}$ in the interval $k_{0} \leq k \leq k_{\max }$. We are interested in sparse networks with finite mean degree $\bar{k} \equiv \int d k k P_{\operatorname{deg}}(k)$. Hence, the degree distribution exponent is assumed to be larger than $2, \gamma>2$.

The node with the maximum degree $k_{\max }$ is called the $h u b$. While the lower bound $k_{0}$ is a constant, the maximum degree $k_{\max }$ scales with the network size $L$. It can be estimated from the condition $L \int_{k_{\max }}^{\infty} P_{\operatorname{deg}}(k) d k=1$, which yields that [15]

$$
k_{\max } \sim L^{\beta} \text { with } \beta=\frac{1}{\gamma-1} .
$$

Some SF networks having an explicit cutoff for $k_{\max }$ may have a different value of $\beta$ [31]. In this work, however, we only 
consider the generic SF networks with $\beta$ given by Eq. $[8$.

For the jumping rate, we take

$$
p_{i}(n)=n^{\delta}
$$

for all nodes with a parameter $\delta \geq 0$. We take

$$
T_{j \leftarrow i}= \begin{cases}\frac{1}{k_{i}}, & \text { if } i \text { and } j \text { are linked } \\ 0 & , \text { otherwise }\end{cases}
$$

for the hopping probability ( $k_{i}$ is the degree of a node $i$ ). That is to say, a particle out of $i$ hops to one of its $k_{i}$ neighbors selected at random.

First, one needs to solve the random walk problem in Eq. (4) for the stationary state probability $P_{\text {stat }}(\mathbf{n})$. The random walk problem with the hopping probability (10) was studied in Ref. [17], from which we find that [30]

$$
\omega_{i}=k_{i} .
$$

Inserting $p(n)$ and $\left\{\omega_{i}\right\}$ into Eq. [3], one finds that

$$
f_{i}(n)=\prod_{l=1}^{n}\left(\frac{k_{i}}{l^{\delta}}\right)=\frac{k_{i}^{n}}{(n !)^{\delta}},
$$

which further yields from Eq. (6) that

$$
F_{i}(z)=\sum_{n=0}^{\infty} \frac{\left(z k_{i}\right)^{n}}{(n !)^{\delta}} .
$$

Hence, the mean occupation number in the stationary state is given from Eq. (5) by

$$
m_{i}(z)=\left.x \frac{\partial \ln \mathcal{F}_{\delta}(x)}{\partial x}\right|_{x=z k_{i}},
$$

where

$$
\mathcal{F}_{\delta}(x) \equiv \sum_{n=0}^{\infty} \frac{x^{n}}{(n !)^{\delta}}
$$

The fugacity $z$ has to be determined from the self-consistent equation.

We add a few remarks on the stationary state probability distribution. Although the jumping rate is the same at all nodes, the probability factor $f_{i}(n)$ in Eq. (12) has the node dependence due to the structural inhomogeneity of underlying networks. On the other hand, consider a ZRP in periodic lattices with disordered jumping rates $p_{i}^{\prime}(n)=n^{\delta} / k_{i}$ and with nearest neighbor particle hoppings. It is evident that the two models share the same stationary state. That is, the structure inhomogeneity plays the same role as the quenched disorder in the jumping rate. At $\delta=0$, in particular, the corresponding ZRP in periodic lattices is characterized by the disordered jumping rate $p^{\prime}=1 / k$ whose distribution is given by

$$
P_{\text {jump }}\left(p^{\prime}\right)=P_{\mathrm{deg}}(k)\left|\frac{d k}{d p^{\prime}}\right| \sim p^{(\gamma-2)} .
$$

Such a problem has been studied in the context of a disordered ZRP and a disordered exclusion process in one dimension [8, 9, 10, 11]. In those studies, the jumping rate distribution is taken to be $P_{\text {jump }}\left(c<p^{\prime}<1\right) \sim\left(p^{\prime}-c\right)^{v}$ with the model parameter $v$ and $c$ for the minimum jumping rate. The present model at $\delta=0$ coincides with that with $c=0$ and $v=\gamma-2$.

It is noteworthy that the mean occupation number in the stationary state is solely determined by the degree, which is a local quantity, and independent of any other characteristics of a network. This, however, is not the case for the relaxation dynamics studied in Sec.IV

At $\delta=1$, the particle jumping rate is directly proportional to the occupation number. Particles move independently and the system reduces to a non-interacting system of $N$ random walkers. So, the stationary state occupation number distribution becomes proportional to the single walker distribution in Eq. (11), $m_{i} \propto \omega_{i}=k_{i}$. This solution is also obtained from the general formula in Eq. (14). Since $\mathcal{F}_{1}(x)=e^{x}, m_{i}(z)=z k_{i}$, which is proportional to the degree as expected.

For general values of $\delta$, the solution is non-trivial. We will solve the problem for $\delta=0$ and $\delta \neq 0$ separately.

\section{A. $\delta=0$ case}

At $\delta=0$, the infinite series for $\mathcal{F}_{\delta}(x)$ has a closed form expression $\mathcal{F}_{0}(x)=1 /(1-x)$, which yields

$$
m_{i}(z)=\frac{z k_{i}}{1-z k_{i}} .
$$

The fugacity is in the range $z<z_{c}=1 / k_{\max } \ll 1$.

Note that $m_{i}(z)$ is divergent for the hub as $z$ approaches $z_{c}$. So, it is convenient to decompose the self-consistent equation as $\rho=\rho_{s}+\rho_{n}$ where

$$
\rho_{s}=\frac{m_{\mathrm{hub}}}{L}=\frac{1}{L} \frac{z k_{\max }}{1-z k_{\max }}
$$

is the particle density at the hub and

$$
\rho_{n}=\frac{1}{L} \sum_{i \neq \text { hub }} \frac{z k_{i}}{1-z k_{i}}
$$

is the remaining density. For large $L$, one can evaluate $\rho_{n}$ as

$$
\rho_{n}=\int_{k_{0}}^{k_{\max }} d k \frac{P_{\mathrm{deg}}(k) z k}{1-z k}=z^{\gamma-1} \int_{k_{0} z}^{k_{\max } z} d x \frac{x^{1-\gamma}}{(1-x)} .
$$

The integrand becomes singular at both integral endpoints as $z k_{\max } \rightarrow 1$ and $z k_{0} \rightarrow 0$. Evaluating the singular parts, we obtain that $\rho_{n}=O(z)+O\left(z^{\gamma-1} \ln \left(1-z k_{\max }\right)\right)$, where the first (second) term is a contribution near the lower (upper) endpoint [32]. Since $z<1 / k_{\max } \sim L^{-\beta} \ll 1$ and $\gamma>2$, $\rho_{n}$ vanishes in the large $L$ limit. Therefore we conclude that the whole fraction of particle should be condensed into the hub [complete condensation],

$$
\rho_{s}=m_{\mathrm{hub}} / L=\rho .
$$


The fugacity is then given by

$$
z=k_{\max }^{-1} /(1+1 /(\rho L)) \sim L^{-\beta}(1-1 /(\rho L)) .
$$

So the mean occupation number at other nodes with $k_{i} \ll k_{\max }$ is given as

$$
m_{i \neq \mathrm{hub}} \simeq z k_{i} \simeq k_{i} / k_{\max } \sim L^{-\beta} k_{i}
$$

which is proportional to the degree with the vanishing coefficient.

\section{B. $\delta>0$ case}

For $\delta>0$, the infinite series in Eq. (15) does not have a closed form expression except for $\mathcal{F}_{\delta=1}(x)=e^{x}$. We approximate the series in the following way. Putting the summand as $x^{n} /(n !)^{\delta} \equiv \exp [g(n)]$ and using the Stirling formula, one obtains that

$$
g(n) \simeq n \ln x-\delta\left\{\frac{1}{2} \ln (2 \pi n)+n \ln n-n\right\} .
$$

This function is peaked at $n=n_{0}$ and behaves near $n \simeq n_{0}$ as

$$
g(n) \simeq g\left(n_{0}\right)+\frac{1}{2} g^{\prime \prime}\left(n_{0}\right)\left(n-n_{0}\right)^{2}+\cdots .
$$

The peak position is easily found to be $n_{0} \simeq x^{1 / \delta}$ from the condition $g^{\prime}\left(n_{0}\right)=0$, which yields $g^{\prime \prime}\left(n_{0}\right)=-\delta / n_{0}+O\left(n_{0}^{-2}\right)$. We then approximate the series with the Gaussian integral as

$$
\begin{aligned}
\mathcal{F}_{\delta>0}(x) & \simeq e^{g\left(n_{0}\right)} \int_{-n_{0}}^{\infty} d n^{\prime} \exp \left[g^{\prime \prime}\left(n_{0}\right) n^{\prime 2} / 2\right] \\
& \simeq e^{g\left(n_{0}\right)} \int_{-\infty}^{\infty} d n^{\prime} \exp \left[g^{\prime \prime}\left(n_{0}\right) n^{\prime 2}\right] \\
& \simeq \frac{1}{\sqrt{\delta}}\left(2 \pi x^{1 / \delta}\right)^{(1-\delta) / 2} \exp \left(\delta x^{1 / \delta}\right) .
\end{aligned}
$$

In the second step, we extend the integration interval. This approximation is valid when $\left|g^{\prime \prime}\left(n_{0}\right)\right| n_{0}^{2}=\delta x^{1 / \delta} \gtrsim 1$, that is $x \gtrsim(1 / \delta)^{\delta}=O(1)$. For $x \ll 1$, one can simply approximate $\mathcal{F}_{\delta}(x)$ with a few lowest order terms as $\mathcal{F}_{\delta}(x)=1+x+O\left(x^{2}\right)$.

Note that the approximation in Eq. (23) coincides with the exact result at $\delta=1$. We have tested its validity at other values of $\delta$ numerically. Figure 1 shows that the approximation is very good unless $x \simeq 0$.

Using the approximation for $\mathcal{F}_{\delta}(x)$, the mean occupation number is given by

$$
m_{i} \simeq \begin{cases}z k_{i} & \text { for } \quad z k_{i} \ll 1 \\ \left(z k_{i}\right)^{1 / \delta} & \text { for } \quad z k_{i} \gtrsim 1\end{cases}
$$

The mean occupation number of a node increases monotonically with its degree. So we expect that $m_{\text {hub }} \gtrsim 1$ and that $z k_{\max } \gtrsim 1$. On the other hand, for a node with the minimum degree $k_{0}$, one may have $z k_{0} \ll 1$ or $z k_{0} \gtrsim 1$ depending on the magnitude of $z$. Hence, one must consider the following two cases separately.
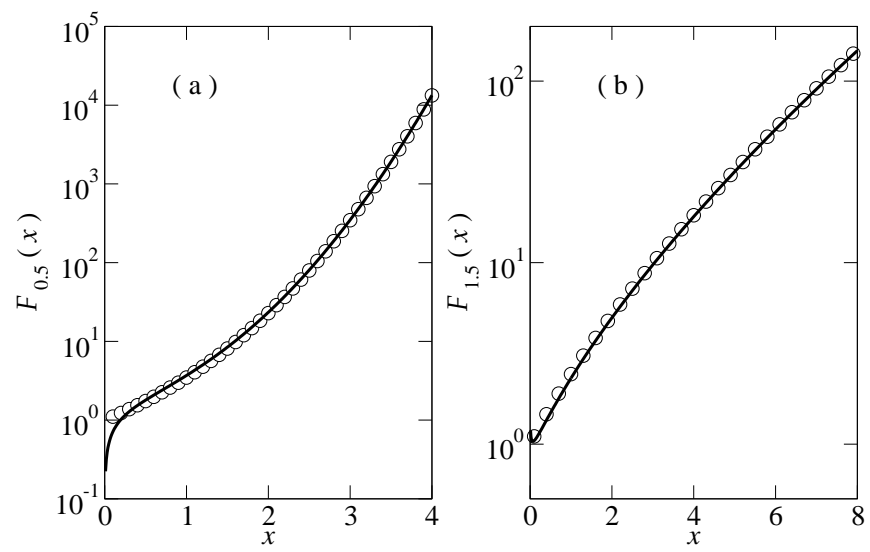

FIG. 1: Comparison of $\mathcal{F}_{\delta}(x)$ (circular symbols) defined in Eq. 15 and the approximation (solid line) in Eq. 23 at $\delta=0.5$ (a) and 1.5 (b).

(i) Firstly, let us assume that the fugacity is in such a range that $z k_{i} \gtrsim 1$ for all nodes. This is the case when $z$ is a nonvanishing constant in the large $L$ limit. Then the occupation number is given by $m_{i} \simeq\left(k_{i} z\right)^{1 / \delta}$ for all nodes, and the selfconsistent equation has the solution $z=\rho^{\delta} /\left(\overline{k^{1 / \delta}}\right)^{\delta}$ where $\overline{k^{1 / \delta}} \equiv \int_{k_{0}}^{k_{\max }} d k k^{1 / \delta} P_{\operatorname{deg}}(k)$. It could be a non-vanishing constant only when $\overline{k^{1 / \delta}} \sim \int_{k_{0}}^{k_{\max }} d k k^{-(\gamma-1 / \delta)}$ remains finite in the large $L$ limit. It imposes that $\delta>\delta_{c}$ with

$$
\delta_{c}=\frac{1}{\gamma-1}
$$

In this regime, we find that

$$
m_{i} \simeq c k_{i}^{1 / \delta}
$$

for all nodes with a constant $c=\rho /\left(\overline{k^{1 / \delta}}\right)$. The occupation number at the hub with the degree $k_{\max } \sim L^{\beta}$ scales as $m_{\text {hub }} \sim L^{\beta / \delta}$. Since $\beta=1 /(\gamma-1)$ and $\delta>\delta_{c}=1 /(\gamma-1)$, the occupation number at the hub scales sub-linearly in $L$. Therefore, the condensation does not occur when $\delta>\delta_{c}$.

(ii) Secondly, let us assume that the fugacity $z$ is in such a range that the crossover degree defined as $k_{c} \equiv 1 / z$ scales with the network size $L$ and is in the interval $k_{0} \ll k_{c} \ll k_{\max }$. Then, the self-consistent equation becomes $\rho=\rho_{n}+\rho_{s}$ where

$$
\rho_{n}=k_{c}^{-1} \int_{k_{0}}^{k_{c}} d k k P_{\operatorname{deg}}(k)
$$

is the density at small-degree nodes with $k<k_{c}$ and

$$
\rho_{s}=k_{c}^{-1 / \delta} \int_{k_{c}}^{k_{\max }} d k k^{1 / \delta} P_{\mathrm{deg}}(k)
$$

is the density at large-degree nodes with $k>k_{c}$. The integral part in Eq. 27) is smaller than the mean degree $\bar{k}$ that is finite $(\gamma>2)$. So, $\rho_{n}$ vanishes as $\sim k_{c}^{-1}$ in the large $L$ 
limit, which yields that $\rho_{s}=\rho$. In order to have a finite value of $\rho_{s}, \int_{k_{c}}^{k_{\max }} d k k^{1 / \delta-\gamma}$ should be divergent, which yields that $\delta \leq \delta_{c}=1 /(\gamma-1)$. And it should be of the same order as $k_{c}^{-1 / \delta}$, which yields

$$
k_{c} \sim \begin{cases}{\left[\ln k_{\max }\right]^{\delta_{c}},} & \text { for } \quad \delta=\delta_{c} \\ {\left[k_{\max }\right]^{1-\delta / \delta_{c}},} & \text { for } \delta<\delta_{c}\end{cases}
$$

With the crossover degree $k_{c}=1 / z$, the occupation number distribution is given by Eq. 24, which can be summarized with the scaling form

$$
m_{i}=G_{\delta}\left(k_{i} / k_{c}\right)
$$

with the scaling function $G_{\delta}(y)$ that behaves as $G_{\delta}(y \ll 1) \sim y$ and $G_{\delta}(y \gtrsim 1) \sim y^{1 / \delta}$.

We add a few remarks on the result in Eq. (30). Nodes with $k_{i}<k_{c}$ covers the whole fraction of nodes in a network since $\int_{k_{c}}^{k_{\max }} d k P_{\operatorname{deg}}(k) \sim k_{c}^{-(\gamma-1)}$ vanishes in the $L \rightarrow \infty$ limit. However, the total density of particles on those nodes, given by $\rho_{n} \sim k_{c}^{-1}$, vanishes in the thermodynamic limit. Therefore, the whole fraction of particles are condensed onto the vanishingly small fraction of nodes with $k_{i}>k_{c}$. For those nodes, the occupation number is given by $m_{i} \simeq\left(k_{i} / k_{c}\right)^{1 / \delta} \sim$ $\left(k_{\max } / k_{c}\right)^{1 / \delta}\left(k_{i} / k_{\max }\right)^{1 / \delta}$. It has the overall $L$ dependence as $\left(k_{\max } / k_{c}\right)^{1 / \delta} \sim L^{\beta / \delta_{c}} \sim L$ for $\delta<\delta_{c}$ and $\sim L / \ln L$ for $\delta=\delta_{c}$. That is, the occupation number scales linearly with the system size $L$ for $\delta<\delta_{c}$. One can also find that the scaling form in Eq. (30) can be applied to the case of $\delta=0$ studied in the previous subsection with $k_{c}=k_{\max }$. Therefore, we conclude that there is the complete condensation for $\delta<\delta_{c}$. At the threshold $\delta=\delta_{c}$, the logarithmic correction appears.

As explained earlier, the stationary state of the present model is equivalent to that of the disordered ZRP on periodic lattices with the jumping rate $p_{i}^{\prime}(n)=n^{\delta} / k_{i}$. The disordered ZRP with $n$-independent jumping rates, which corresponds to the $\delta=0$ case, has been studied in Refs. [8, 9, 10, 11]. Those studies show that the condensation is triggered by the node with the slowest hopping rate and the complete condensation occurs when the slowest jumping rate vanishes [8, 9, 10, 11]. Generalizing the results, one can understand our phase diagram intuitively. The slowest hopping rate node corresponds to the hub with the maximum degree $k_{\max } \sim L^{\beta}$. Assuming that the node is occupied macroscopically, one finds that the jumping rate is given by $p^{\prime}=n^{\delta} / k_{i} \sim L^{\delta-\beta}$. It vanishes when $\delta<\beta=1 /(\delta-1)$, which coincides with the phase boundary.

Summarizing the results, we present the stationary state phase diagram of the ZRP on SF networks in Fig. 2] The region I corresponds to the uncondensed phase, where the occupation number is given by Eq. (26). The region II corresponds to the condensed phase, where the occupation number is given by Eq. (30). The phase boundary is given by $\delta=\delta_{c}$ with Eq. (25). The phase diagram suggests that the condensation also occurs in random networks $(\gamma=\infty)$ at $\delta=0$, which is observed in a numerical study [33]. The occupation number distribution in each phase is sketched in Fig. 2

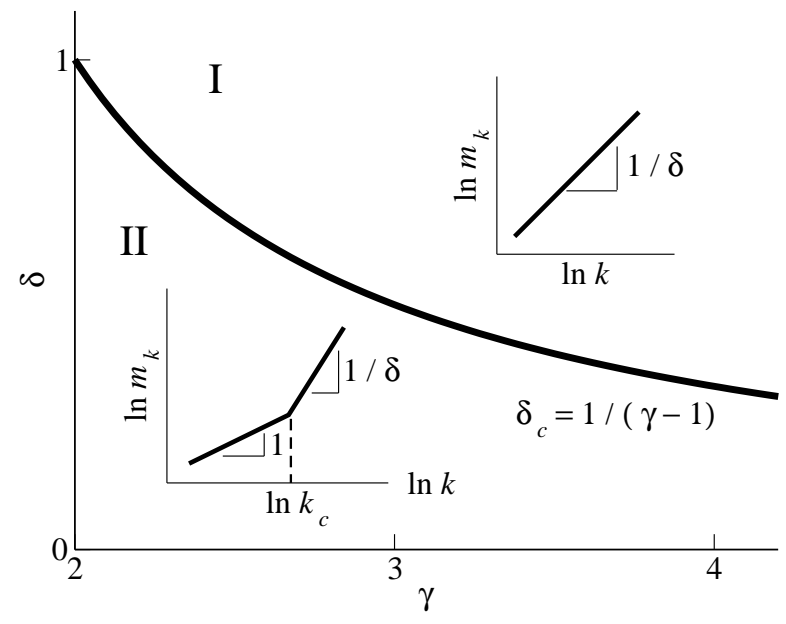

FIG. 2: Stationary state phase diagram. The region I and II corresponds to the uncondensed and condensed phase, respectively.

\section{RELAXATION DYNAMICS}

The preceding analysis shows that the occupation number distribution in the stationary state is solely determined by the degree distribution $P_{\mathrm{deg}}(k)$ being independent of any other characteristics of underlying networks. However, the relaxation dynamics may be affected by the structure of underlying networks. We are interested in the relaxation dynamics in the condensation phase. Unlike the stationary state property, an exact theory is not available for the dynamical property of the ZRP. We rely on Monte Carlo simulations for the study and provide a scaling argument to understand dynamical scaling properties.

We describe our Monte Carlo simulation algorithm. Initially at time $t=0, N$ particles are distributed uniformly and randomly on a network of $L$ nodes. At each step, a particle is selected at random. Let $i$ be its residing site and $n_{i}$ be the occupation number there. Then, with the probability $p\left(n_{i}\right) / n_{i}=n_{i}^{\delta-1}$, the particle jumps out of $i$ and hops to one of its neighboring nodes. With the probability $1-p\left(n_{i}\right) / n_{i}$, the selected particle does not move. The time is measured in unit of one Monte Carlo sweep consisting of $N$ trials. Note that the particle-based dynamics with the modified jumping probability $p(n) / n$ is equivalent to the original node-based dynamics with the jumping rate $p(n)$.

As for SF networks, we use the Dorogovtsev-MendesSamukhin model [34] that is a generalization of the BarabásiAlbert model [35]. With the model, one can generate networks of tree structure or looped structure with arbitrary values of the degree distribution exponent $\gamma$.

In order to get an insight into the nature of the relaxation dynamics, we have observed the time evolution of the mean occupation number distribution. The Monte Carlo simulation data are presented in Fig. 3] (a). They are obtained on the looped SF network of $L=10^{4}$ nodes with $\gamma=3\left(\delta_{c}=1 / 2\right)$. The particle density is taken to be $\rho=1$. We take the jumping rate $p(n)=n^{\delta}$ with $\delta=0.2$ so that the system is in the condensation phase. The plotted quantity $m_{k}$ is the ensemble 

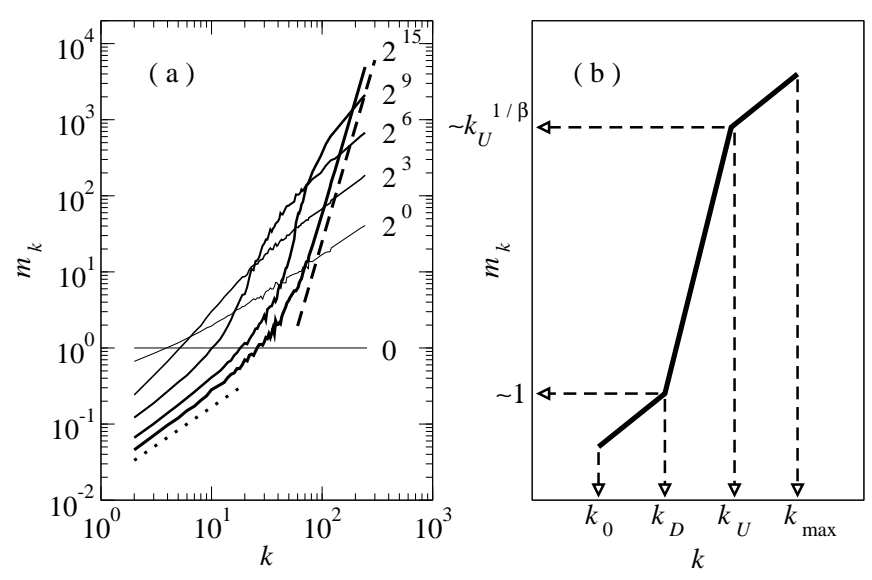

FIG. 3: (a) The occupation number distribution $m_{k}$ at $0 \leq t \leq 2^{15}$. The dotted (dashed) line has the slope $1(1 / \delta=5)$. (b) Schematic plot of $m_{k}$ vs $k$ in the log-log scale in the transient time.

average of the occupation number $n_{i}$ averaged over nodes with the degree $k_{i}=k$.

The data show how the distribution, starting from the uniform distribution at $t=0$, approaches the stationary distribution. One can see that the initial uniform distribution evolves very quickly (at $t=1$ ) into a linear distribution $m_{k} \sim k$. This is due to the random walk nature of the initial dynamics. The initial uniform distribution implies that the jumping probability is the same for all particles at $t=0$. So they behave as non-interacting random walkers, which has the distribution $m_{k} \sim k$. After that, the distribution evolves further and approaches the stationary distribution. The MC data shows that the system has already reached the stationary distribution given in Eq. (30) at $t=2^{15}$.

In the transient period, we find that there are three different regimes that are separated by two time-dependent degree scales denoted by $k_{D}$ and $k_{U}$. It turns out that the occupation number distribution behaves in each regime as

$$
m_{k} \sim\left\{\begin{array}{lll}
k^{1} & \text { for } & k \lesssim k_{D} \\
k^{1 / \delta} & \text { for } & k_{D} \lesssim k \lesssim k_{U} \\
k^{1} & \text { for } & k_{U} \lesssim k
\end{array}\right.
$$

We illustrate the behavior with the schematic plot in Fig. 33 (b). The degree scales grow in time and approach $k_{D} \rightarrow k_{c}$ with the crossover degree $k_{c}$ given in Eq. (29) and $k_{U} \rightarrow k_{\max }$ in the stationary state limit. Furthermore, the behavior of $m_{k}$ for $k<$ $k_{U}$ is identical to the stationary state distribution in Eq. (30) with $k_{D}$ and $k_{U}$ playing the role of $k_{c}$ and $k_{\max }$, respectively. For nodes with $k>k_{U}, m_{k} \propto k$. They are still in the random walk regime.

These observations lead us to conjecture that the relaxation dynamics has a hierarchical nature: A sub-network of smalldegree nodes with $k<k_{U}(t)$ is equilibrated first and largedegree nodes become equilibrated as the degree scale $k_{U}(t)$ grows in time. In a transient time $t$, those nodes with $k<$ $k_{U}(t)$ reach the stationary state of a smaller network of size $L^{\prime}(t) \sim k_{U}(t)^{1 / \beta}$ with $\beta=1 /(\gamma-1)$. The other degree scale $k_{D}(t)$ plays the role of the crossover degree scale in the smaller

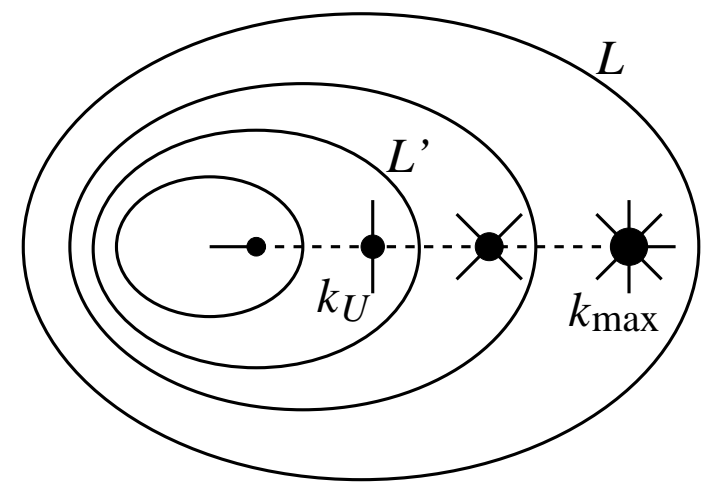

FIG. 4: Illustration the hierarchical relaxation dynamics. Up to an intermediate time step $t$, the subnetwork of $L^{\prime}(t)$ nodes and with the largest degree $k_{U}(t) \sim L^{\prime}(t)^{\beta}$ reaches the stationary state. The equilibrated area expands as $k_{U}$ increases until it reaches $k_{\max }$.

network, that is,

$$
k_{D} \sim \begin{cases}k_{U}^{1-\delta / \delta_{c}} & \text { for } \delta<\delta_{c} \\ \left(\ln k_{U}\right)^{\delta_{c}} & \text { for } \delta=\delta_{c}\end{cases}
$$

following from Eq. 29]. This hierarchical nature is illustrated in Fig. 4

The conjecture predicts that the relaxation dynamics is determined by the temporal evolution of the degree scale $k_{U}(t)$. We develop a scaling theory for $k_{U}(t)$. Suppose that the system is equilibrated up to a sub-network of $L^{\prime}$ nodes with the maximum degree $k_{U} \sim L^{\prime \beta}$ with $\beta=1 /(\gamma-1)$. The node with the maximum degree will be called a temporary hub. In order to proceed to the next hierarchy, particles should be transferred to higher degree nodes. We will assume that particle transfers from the temporary hub to the next hub dominate the relaxation time scale, which will be confirmed numerically shortly. Since the sub-network is in the stationary state, there are $n \sim L^{\prime} \sim k_{U}^{1 / \beta}$ particles at the temporary hub. During the unit time interval, $\Delta n=p(n) \sim L^{\prime \delta}$ particles jump out of the temporary hub and perform the random walk motions. They may wander within the sub-network and return to the temporary hub, or escape to the higher degree node in the next hierarchy level. Let $P_{\text {esc }}\left(L^{\prime}\right)$ be the escape probability for the latter process. Then, we obtain the following scaling relation

$$
t \sim \frac{1}{P_{\mathrm{esc}}\left(L^{\prime}\right)} \times\left(\frac{n}{\Delta n}\right) \sim \frac{1}{P_{\mathrm{esc}}\left(L^{\prime}\right)} \times L^{\prime 1-\delta} .
$$

The escape probability depends on the structure of the underlying networks. Suppose that the underlying SF network has a tree structure with no loop. On a tree a path from one node to another is unique. So, particles can escape from the temporary hub only when they hop along the link to the right direction toward the next hub among $k_{U}$ links. Hence the escape probability is given by

$$
P_{\mathrm{esc}}\left(L^{\prime}\right) \simeq k_{U}^{-1} \sim L^{\prime-\beta}
$$

for a tree network. On the other hand, in general networks with many loops, there are multiple pathways between two 
nodes. Hence, we expect that the escape probability does not scale algebraically with $L^{\prime}$ or $k_{U}$, that is,

$$
P_{\mathrm{esc}}\left(L^{\prime}\right) \sim L^{\prime 0} \sim k_{U}^{0}
$$

for a looped network. Combining Eqs. (33) (34), and (35), we obtain that

$$
L^{\prime} \sim t^{1 /(1+\beta-\delta)} \text { or } k_{U} \sim t^{\beta /(1+\beta-\delta)}
$$

for a tree network and

$$
L^{\prime} \sim t^{1 /(1-\delta)} \text { or } k_{U} \sim t^{\beta /(1-\delta)}
$$

for a looped network. The scaling behavior of $k_{D}$ is obtained from Eq. (32).

The system reaches the global stationary state when $L^{\prime}$ becomes equal to the network size $L$ or, equivalently, $k_{U}$ becomes equal to $k_{\max } \sim L^{\beta}$. The power-law scaling of $L^{\prime}$ and $k_{U}$ implies that the relaxation time also scales algebraically as

$$
\tau \sim L^{z}
$$

The dynamic exponent $z$ depends on the global structure of underlying networks and is given by

$$
z= \begin{cases}1+\beta-\delta, & \text { for a tree network } \\ 1-\delta, & \text { for a looped network }\end{cases}
$$

At the transition $\delta=\delta_{c}$, there is a logarithmic correction to the power-law scaling.

We add a remark on the result in Eq. 39. As explained in Sec. III our model at $\delta=0$ has the same stationary state as the disordered ZRP in periodic lattices with the jumping rate distribution $P_{\text {jump }}\left(p^{\prime}\right)=p^{\prime \nu}$ with $v=\gamma-2$. The relaxation dynamics of the ZRP has also been studied in Refs. [10, 11]. It is known to have $z=(v+2) /(v+1)$ with the biased hopping in one dimension lattices. Interestingly, it coincides with the result for the ZRP on tree structure SF networks $(\beta=1 /(\gamma-$ $1))$. In the disordered ZRP the relaxation proceeds via a lateral growth of a domain within which particles are condensed on to the node with the slowest jumping rate. This mechanism is identical to the hierarchical relaxation in the SF tree networks.

We verify numerically the analytic results. We have performed the Monte Carlo simulations on SF networks with various values of $\gamma$ and with several values of $\delta$. In order to study the structure dependent relaxation dynamics, we have performed the simulations both on tree networks and looped networks.

According to the scaling argument, the degree scale $k_{U}(t)$ separating the second and the third region in Eq. (32) scales as $k_{U}(t) \sim t^{\beta / z}$. And the occupation number at the node with $k=$ $k_{U}$ scales as $m_{k_{U}} \sim k_{U}^{1 / \beta} \sim t^{1 / z}$. Hence, the existence of $k_{U}$ can be verified from the scaling plot of $m_{k}(t) / t^{1 / z}$ against $k / t^{\beta / z}$ at transient times $t \ll \tau$. In Fig. 5 (a), we present the data for the ZRP with $\delta=0.2$ on a tree-structured SF network of $L=10^{5}$ nodes and with $\gamma=4$. All data at different $t$ collapse very well near $k \sim t^{\beta / z}$ with the proposed value of the dynamic exponent $z=1+\beta-\delta$. Figure 5 (b) shows the data of the ZRP
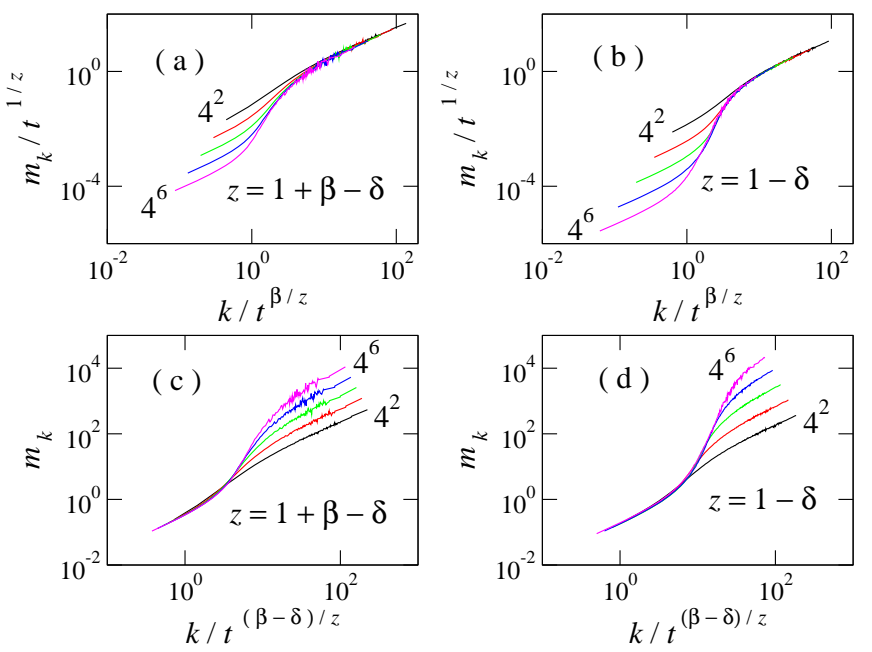

FIG. 5: (color online) Data collapse analysis of Monte Carlo data of the ZRP with $\delta=0.2$ on SF networks of $L=10^{5}$ nodes and with $\gamma=$ 4. Data in (a) and (c) are from the SF networks with the tree structure, while those in (b) and (d) are from the looped SF networks. The data are measured in the transient period at $t=4^{2}$ (black), $4^{3}$ (red), $\cdots$, $4^{6}$ (magenta).

with the same value of $\delta=0.2$ on a looped SF network with the same value of $\gamma=4\left(\delta_{c}=\beta=1 / 3\right)$. We also observe the perfect data collapse near $k \sim t^{\beta / z}$ with the proposed value of the dynamic exponent $z=1-\delta$. These support the structure dependent dynamic scaling of the $k_{U}$.

Our scaling argument also predicts the existence of the degree scale $k_{D}(t) \sim k_{U}^{1-\delta / \delta_{c}}$ which plays the role of the crossover degree $k_{c}$ for nodes with $k<k_{U}(t)$. The scaling $k_{U}(t) \sim t^{\beta / z}$ implies that $k_{D} \sim t^{\left(\beta-\delta \beta / \delta_{c}\right) / z}$. Note that $\beta=\delta_{c}=1 /(\gamma-1)$. Hence, it can be verified with the scaling plot of $m_{k}$ against $k / t^{(\beta-\delta) / z}$. Since the occupation number at the crossover degree is a constant, the occupation number need not be rescaled. We present the scaling plots in Fig.5(c) and (d) for a tree SF network and a looped SF network with the corresponding dynamic exponent, respectively. Data from different times $t$ collapse very well near $k \sim t^{(\beta-\delta) / z}$, which supports the analytic result. We have also performed the data collapse analysis at several other values of $\gamma$ and $\delta$, and obtained the consistent result with the analytic result.

In addition to the data collapse analysis, we also measure the dynamic exponent $z$ through a temporal scaling behavior of $m_{\text {hub }}$. The scaling picture predicts that nodes with $k<k_{U}$ are equilibrated while nodes with $k>k_{U}$ are in the random walk regime with $m_{k} \propto k$. So the occupation number at the hub scales as $m_{\text {hub }} \sim k_{U}^{1 / \beta} \times\left(k_{\max } / k_{U}\right)$. Since $k_{U} \sim t^{\beta / z}$ in the transient times $t \ll \tau$, the occupation number at the hub shows the temporal scaling

$$
m_{\mathrm{hub}} \sim t^{(1-\beta) / z}
$$

From the temporal scaling of $m_{\text {hub }}$, we obtained the values of the dynamic exponent $z$ at different values of $\gamma$ and $\delta$ on SF networks of size $L=10^{5}$ of the tree and looped structure. We present the result in Fig. 6 All the results are consistent with 


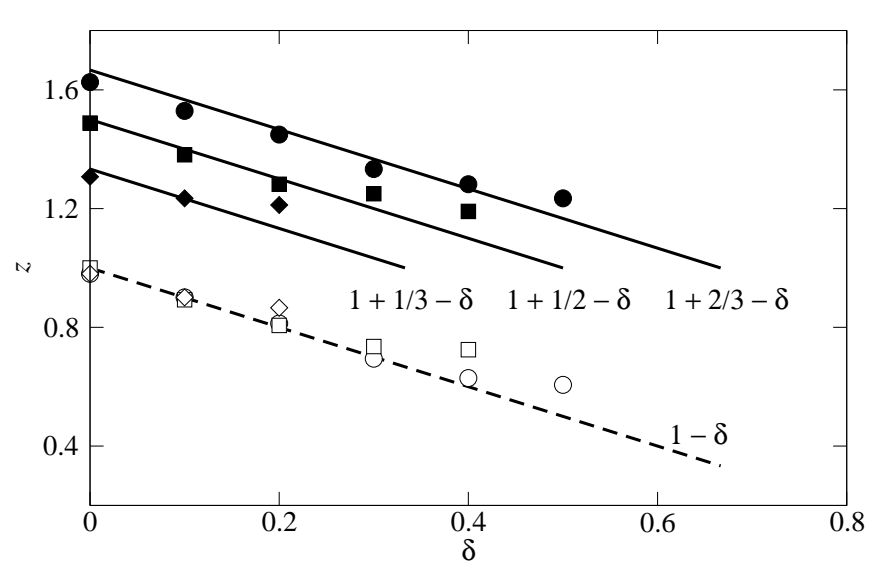

FIG. 6: Dynamic exponent of tree (filled symbols) structure SF networks and looped (open symbols) structure SF networks. Each symbol stands for $\gamma=5 / 2$ (circle), $\gamma=3$ (square), and $\gamma=4$ (diamond). The solid and dashed lines represent the analytic result in Eq. 39 for tree and looped networks, respectively.

the analytic results in Eq. 39). Approaching $\delta=\delta_{c}=1 /(\gamma-$ $1)$, the numerical results seem to deviate from the analytic predictions. It is attributed to strong finite size effects near $\delta=\delta_{c}$ for we obtained better results with larger system sizes.

The numerical analysis supports the conjecture of the hierarchical relaxation and the scaling argument for the structuredependent dynamic exponent $z$ in the condensed phase with $\delta \leq \delta_{c}$. Our result shows that the dynamical property depends on the global structure of the underlying networks, although the stationary state property does not.

\section{SUMMARY}

We have studied the stationary and dynamical properties of the ZRP with the jumping rate $p(n)=n^{\delta}$ on SF networks with the degree distribution exponent $\gamma$. The stationary state property is determined by the degree distribution of underlying networks. The model displays the stationary state condensation transition and the phase diagram is given in Fig. 2 The phase transition is driven by the structural disorder in underlying scale-free networks. It is distinct from the Bose-Einstein type condensation transition found in systems with disordered jumping rates, and from the spontaneous symmetry breaking transition found in systems on periodic lattices. In the condensed phase $\delta \leq \delta_{c}=1 /(\gamma-1)$, the mean occupation number distribution follows the scaling form of Eq. (30). It shows that the whole fraction of particles are concentrated on higher degree nodes with $k>k_{c}$ given in Eq. 29]. On the other hand, the dynamical property depends also on the global structure of underlying networks. The relaxation dynamics is found to have the hierarchical nature that it proceeds from lower degree nodes toward higher degree nodes. It is characterized by the power-law growth of the degree scale $k_{U}$. From the scaling argument, we show that it grows in time with the different exponents depending on whether underlying networks has a tree or looped structure (see Eqs. (36) and (37)). Consequently, the dynamic exponent for the relaxation time $\tau \sim L^{z}$ also depends on the global structure of networks (see Eq. (39)). Our result shows that the global network structure is the important factor in understanding the dynamical property of complex networks.

\section{Acknowledgments}

This work was supported by Korea Research Foundation Grant (KRF-2004-041-C00139). The author thanks KIAS for the support during the visit.
[1] M. R. Evans, Braz. J. Phys. 30, 42 (2000).

[2] M. R. Evans, Y. Kafri, H. M. Koduvely, and D. Mukamel, Phys. Rev. Lett. 80, 425 (1998).

[3] P. F. Arndt, T. Heinzel, and V. Rittenberg, J. Phys. A 31, L45 (1998).

[4] Y. Kafri, E. Levine, D. Mukamel, G.M. Schütz, and J. Török, Phys. Rev. Lett. 89, 035702 (2002).

[5] F. Spitzer, Adv. Math. 5, 246 (1970).

[6] S. Großkinsky, G. M. Schütz, and H. Spohn J. Stat. Phys. 113, 389 (2003).

[7] M. R. Evans and T. Hanney, cond-mat/0501338 (2005).

[8] J. Krug and P. A. Ferrari, J. Phys. A 29, L465 (1996).

[9] M. R. Evans, Europhys. Lett. 36, 13 (1996).

[10] J. Krug, Braz. J. Phys. 30, 97 (2000).

[11] K. Jain and M. Barma, Phys. Rev. Lett. 91, 135701 (2003).

[12] K. Huang, Statistical Mechanics, 2nd ed. (Willey, New York, 1987).

[13] D. J. Watts and S. H. Strogatz, Nature (London) 393, 440 (1998).
[14] R. Albert and A. -L. Barabási, Rev. Mod. Phys. 74, 47 (2002).

[15] S. N. Dorogovtsev and J. F. F. Mendes, Adv. Phys. 51, 1079 (2002).

[16] M. E. J. Newman, SIAM Rev. 45, 167 (2003).

[17] J. D. Noh and H. Rieger, Phys. Rev. Lett. 92, 118701 (2004).

[18] J. D. Noh and H. Rieger, Phys. Rev. E 69, 036111 (2004).

[19] M. Barthelemy, A. Barrat, R. Pastor-Satorras, A. Vespignani, Phys. Rev. Lett. 92, 178701 (2004).

[20] L. K. Gallos and P. Argyrakis, Phys. Rev. Lett. 92, 138301 (2004).

[21] M. Catanzaro, M. Boguñá, and R. Pastor-Satorras, Phys. Rev. E 71, 056104 (2005).

[22] V. Sood and S. Redner, Phys. Rev. Lett. 94, 178701 (2005).

[23] J. D. Noh, G. M. Shim, and H. Lee, Phys. Rev. Lett. 94, 198701 (2005).

[24] M. R. Evans and T. Hanney, J. Phys. A 36, L441 (2003).

[25] M. R. Evans, S. N. Majumdar, and R. K. P. Zia, J. Phys. A 37, L275 (2004).

[26] The exact solution is also available when the jumping rate de- 
pends on the occupation numbers at both departure and destination nodes in a particular way [7].

[27] C. Godrèche, J. Phys. A 36, 6313 (2003).

[28] J. Török, cond-mat/0407567(2004).

[29] S. Großkinsky and T. Hanney, cond-mat/0412593 (2004).

[30] In Ref. [23], the normalized distribution $\omega_{i}=k_{i} /\left(\sum_{j} k_{j}\right)$ was used. However the normalization can be ignored.

[31] M. Boguñá, R. Pastor-Satorras, and A. Vespignani, Eur. Phys. J. B 38, 205 (2004); M. Catanzaro, M. Boguñá, and R. Pastor-
Satorras, Phys. Rev. E 71, 027103 (2005).

[32] In Ref. [23], only the second term was considered. However, this does not change any result.

[33] Y. Kim, unpublished (2005).

[34] S.N. Dorogovtsev, J.F.F. Mendes, and A.N. Samukhin, Phys. Rev. Lett. 85, 4633 (2000).

[35] A.-L. Barabási and R. Albert, Science 286, 509 (1999); A.-L. Barabási, R. Albert, and H. Jeong, Physica A 272, 173 (1999). 